Modeling, Identification and Control, Vol. 35, No. 1, 2014, pp. 21-30, ISSN 1890-1328

\title{
Stiffness Analysis and Optimization of a Co-axial Spherical Parallel Manipulator
}

\author{
Guanglei $\mathrm{Wu}$
}

Department of Mechanical and Manufacturing Engineering, Aalborg University, 9220 Aalborg, Denmark. E-mail: gwu@m-tech.aau.dk

\begin{abstract}
This paper investigates the stiffness characteristics of spherical parallel manipulators. By virtue of singular value decomposition, the $6 \times 6$ dimensionally inhomogeneous Cartesian stiffness matrix is transformed into two homogeneous ones, i.e., the rotational and translational stiffness matrices. The decomposed singular values and the corresponding vectors indicate the directions of high/weak stiffness and the stiffness isotropy for the manipulator at a given configuration. Two indices, one for rotation and the other for translation, are introduced to optimize the manipulator stiffness and to map the stiffness isocontours over the workspace to show an image of the overall stiffness.
\end{abstract}

Keywords: Spherical parallel manipulator, parameter nomalization, singular value decomposition, stiffness optimization

\section{Introduction}

Three Degrees of Freedom (3-DOF) spherical parallel manipulators (SPMs) have been extensively studied, with focus on different aspects, such as kinematic analysis Gosselin and Lavoie (1993); Bonev et al. (2006), singularity analysis Bonev and Gosselin (2005), dexterity Bai et al. (2009), workspace analysis Bulca et al. (1999); Bai (2010) and synthesis Kong and Gosselin (2004). On the other hand, the elastostatic/kinetostatic analysis, particularly, the stiffness, received relatively less attention. Liu et al. (2000) proposed a model by only considering the actuation compliance, in which a global stiffness index similar to global conditioning index (GCI) is proposed for isotropy design of SPM. Combining the structural compliance, Enferadi and Tootoonchi (2011) conducted the stiffness analysis of a 3-RRP SPM on the basis of strain energy and Castigliano's theorem by ignoring the influence of the passive joints and strain energy due to shear forces. Since the SPMs are widely used as orientating devices, these stiffness models are limited to investigate the orientation deformation of the mobile platform. Whereas, when this kind of SPMs are used to develop spherical joints Asada and Granito (1985), the translational deformation of the SPM, namely, the separation between the centers of the mobile and base platforms, could be an important consideration. This paper will investigate the stiffness characteristics for this class of SPMs.

On the assessment of manipulator stiffness, a common problem lies in the dimensionally inhomogeneous matrix, which does not admit a norm to evaluate its stiffness. Gosselin (1990) used the diagonal elements of the stiffness matrix to map the stiffness over the workspace, to represent the pure stiffness in each direction clearly and directly. El-Khasawneh and Ferreira (1999) addressed on the direct eigenvalue problem of the stiffness matrix for a mechanism at a given posture to find the minimum and maximum stiffnesses and their directions. However, the identification of eigenvalues and eigenvectors does not make sense for an inhomogeneous matrix due to the different physical meanings of dimensionally inconsistent entries. Ciblak 


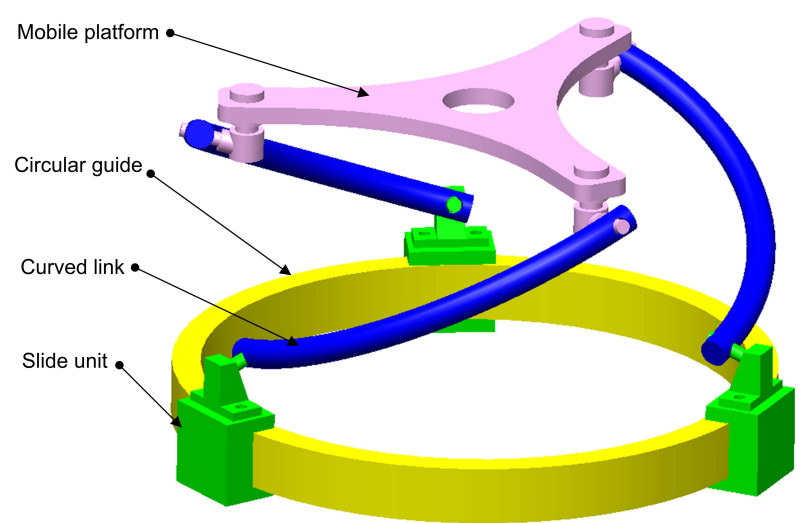

Figure 1: A co-axial spherical parallel manipulator.

and Lipkin (1999) proposed a method of synthesizing a linearly elastic suspension composed of simple translational springs with prescribed stiffness matrix. Ding and Selig (2004) used a finite element model to compute the Cartesian stiffness matrix of a more general suspension (a coiled spring). Dai and Ding (2006) discussed the eigenscrew decomposition of the compliance matrix to find the eigencompliance and eigenscrew for a three-legged platform device. Similarly, Angeles (2010) proposed the eigenforce from the decomposed stiffness matrix to characterize a robot's stiffness. Kövecses and Ebrahimi (2009) proposed a decomposition of the inertia matrix for dynamic analysis by changing variables to make the matrix non-dimensional, which can be applied to the stiffness matrix Taghvaeipour et al. (2012). Henceforth, this approach will be adopted to characterize and to optimize the stiffness of SPMs.

In this paper, the characteristics of the stiffness matrix were analyzed by addressing the matrix inhomogeneity. With the virtual-spring approach Pashkevich et al. (2009), the Cartesian stiffness matrix for the SPMs was developed, which was transformed into homogeneous translational and rotational stiffness matrices by means of singular value decomposition (SVD). Two indices for translational and rotational stiffness were introduced to optimize the manipulator stiffness and reveal the properties. The proposed approach was illustrated with a co-axial SPM Bai (2010); Wu (2012), as displayed in Fig. 1, whose stiffness characteristics at a given configuration were graphically represented by stiffness ellipsoids. The stiffness indices and isotropy were mapped over its workspace to show an image of the overall stiffness.

\section{Manipulator Under Study}

A general spherical parallel manipulator is shown in Fig. 2 for comparison. The SPM consists only of revo-

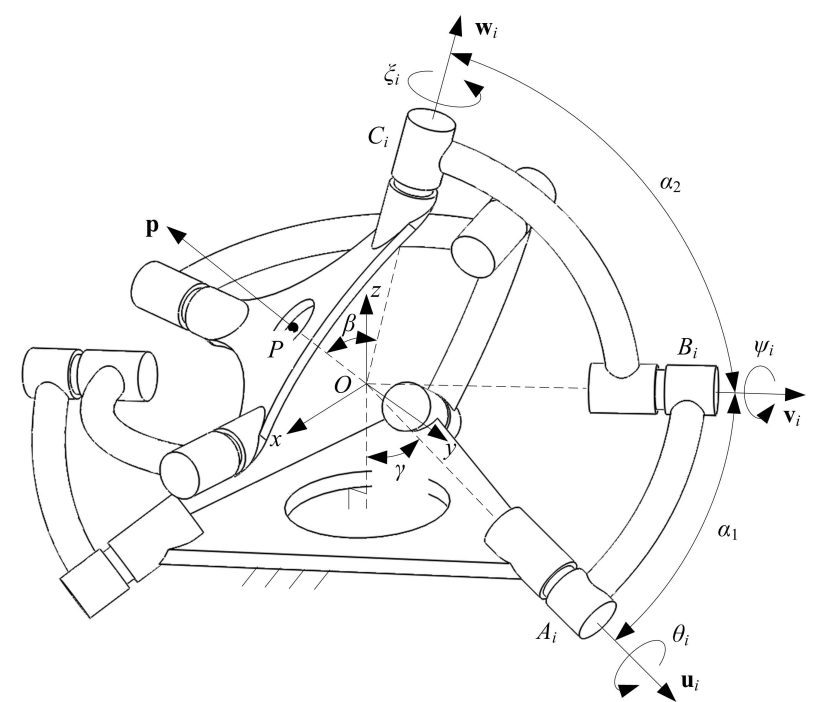

Figure 2: Architecture of a general SPM.

lute joints, whose axes are parallel to the unit vectors $\mathbf{u}_{i}, \mathbf{v}_{i}$, and $\mathbf{w}_{i}, i=1,2,3$, intersecting at a common point, namely, the center of rotation. All three legs have identical structures defined by angles $\alpha_{1}, \alpha_{2}, \beta$ and $\gamma$. The origin $O$ of the reference coordinate system $x y z$ is located at the center of rotation. The $z$ axis is normal to the bottom surface of the base pyramid and points upwards, while the $y$ axis is located in the plane made by $z$ axis and $\mathbf{u}_{1}$. The manipulator shown in Fig. 1 is a special case of $\gamma=0$, which consists only of three curved links connected to a rigid mobile platform (MP) as an end-effector (EE).

Under the prescribed coordinate system, unit vector $\mathbf{u}_{i}$ is derived as

$$
\mathbf{u}_{i}=\left[\begin{array}{lll}
-\sin \eta_{i} \sin \gamma & \cos \eta_{i} \sin \gamma & -\cos \gamma
\end{array}\right]^{T}
$$

where $\eta_{i}=2(i-1) \pi / 3, i=1,2,3$.

Unit vector $\mathbf{v}_{i}$ of the axis of the intermediate revolute joint in the $i$ th leg is expressed as:

$$
\mathbf{v}_{i}=\left[\begin{array}{c}
-\mathrm{s} \eta_{i} \mathrm{~s} \gamma \mathrm{c} \alpha_{1}+\left(\mathrm{c} \eta_{i} \mathrm{~s} \theta_{i}-\mathrm{s} \eta_{i} \mathrm{c} \gamma \mathrm{c} \theta_{i}\right) \mathrm{s} \alpha_{1} \\
\mathrm{c} \eta_{i} \mathrm{~s} \gamma \mathrm{c} \alpha_{1}+\left(\mathrm{s} \eta_{i} \mathrm{~s} \theta_{i}+\mathrm{c} \eta_{i} \mathrm{c} \gamma \mathrm{c} \theta_{i}\right) \mathrm{s} \alpha_{1} \\
-\mathrm{c} \gamma \mathrm{c} \alpha_{1}+\mathrm{s} \gamma \mathrm{c} \theta_{i} \mathrm{~s} \alpha_{1}
\end{array}\right]
$$

Unit vector $\mathbf{w}_{i}$ of the top revolute joint in the $i$ th leg, is a function of the orientation of the mobile platform described as

$$
\mathbf{w}_{i}=\left[\begin{array}{lll}
w_{i x} & w_{i y} & w_{i z}
\end{array}\right]^{T}=\mathbf{Q} \mathbf{w}_{i}^{*}
$$

where $\mathbf{w}_{i}^{*}$ is the unit vector for the axis of the top revolute joint of the $i$ th leg when the mobile platform is at its home configuration, which is given as

$$
\mathbf{w}_{i}^{*}=\left[\begin{array}{lll}
-\sin \eta_{i} \sin \beta & \cos \eta_{i} \sin \beta & \cos \beta
\end{array}\right]^{T}
$$




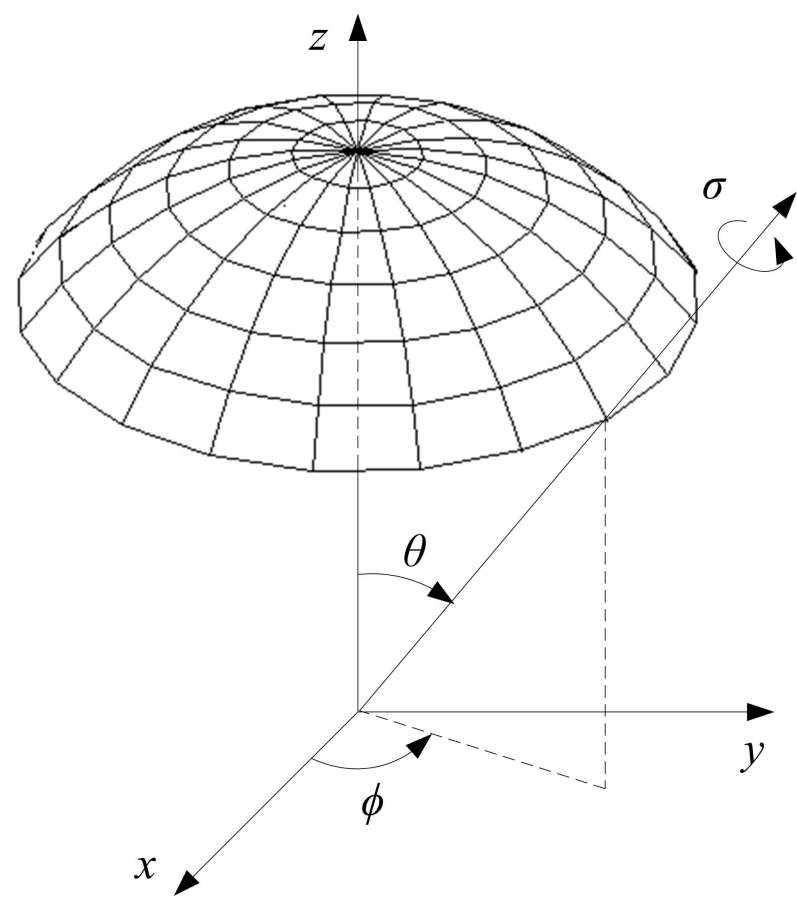

Figure 3: The orientation representation of the azimuth-tilt-torsion angles.

Matrix $\mathbf{Q}$ is the rotation matrix of the mobile platform represented by azimuth-tilt-torsion $(\phi-\theta-\sigma)$ angles Bonev (2008) as shown in Fig. 3, which is kinematically equivalent to the Euler convention $Z-Y-Z$, namely,

$$
\mathbf{Q}=\mathbf{R}_{z}(\phi) \mathbf{R}_{y}(\theta) \mathbf{R}_{z}(\sigma-\phi)
$$

where $\phi \in(-\pi, \pi], \theta \in[0, \pi), \sigma \in(-\pi, \pi]$.

\section{Stiffness Modeling of SPM}

In this work, the virtual spring approach Pashkevich et al. (2009) is adopted to derive the Cartesian stiffness matrix of SPM, of which the details of modeling procedure were presented in Wu et al. (2014). Figure 4(a) represents the flexibility of the single kinematic leg with the virtual springs, of which the two passive joint bearings are supposed to be rigid. Moreover, the joint displacements and the coordinate frames in the joint space of the $i$ th leg are illustrated in Fig. 4(b).

Let the center of rotation be the reference point of the end-effector. Henceforth, the Jacobians of one leg are expressed as below:

$$
\begin{aligned}
& \mathbf{J}_{\theta}^{i}=\left[\begin{array}{llll}
\hat{\$}_{i A} & \hat{\boldsymbol{\$}}_{i 1} & \ldots & \hat{\boldsymbol{\$}}_{i 12}
\end{array}\right] \in \mathbb{R}^{6 \times 13} \\
& \mathbf{J}_{q}^{i}=\left[\begin{array}{ll}
\hat{\$}_{i B} & \hat{\$}_{i C}
\end{array}\right] \in \mathbb{R}^{6 \times 2}
\end{aligned}
$$

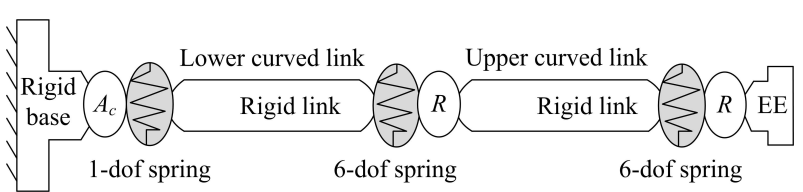

(a)

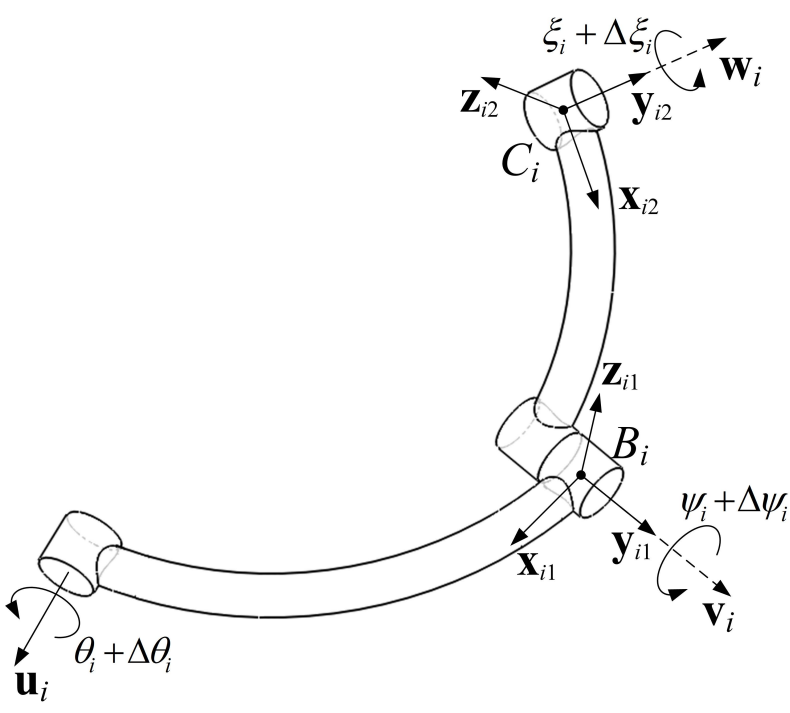

(b)

Figure 4: Flexibilities of one single leg: (a) virtual spring model, where $A_{c}$ stands for the actuator, $R$ for revolute joints; (b) joint displacements in the $i$ th leg in local frames.

with

$$
\begin{aligned}
& \hat{\boldsymbol{\$}}_{i A}=\left[\begin{array}{c}
\mathbf{u}_{i} \\
\mathbf{0}
\end{array}\right], \hat{\boldsymbol{\$}}_{i B}=\left[\begin{array}{c}
\mathbf{v}_{i} \\
\mathbf{0}
\end{array}\right], \hat{\boldsymbol{\$}}_{i C}=\left[\begin{array}{c}
\mathbf{w}_{i} \\
\mathbf{0}
\end{array}\right] \\
& \hat{\boldsymbol{\$}}_{i 1}=\left[\begin{array}{c}
\mathbf{x}_{i 1} \\
\mathbf{b}_{i} \times \mathbf{x}_{i 1}
\end{array}\right], \hat{\boldsymbol{\$}}_{i 2}=\hat{\boldsymbol{\$}}_{i B}, \hat{\boldsymbol{\$}}_{i 3}=\left[\begin{array}{c}
\mathbf{z}_{i 1} \\
\mathbf{b}_{i} \times \mathbf{z}_{i 1}
\end{array}\right] \\
& \hat{\boldsymbol{\$}}_{i 4}=\left[\begin{array}{c}
\mathbf{0} \\
\mathbf{x}_{i 1}
\end{array}\right], \hat{\boldsymbol{\$}}_{i 5}=\left[\begin{array}{c}
\mathbf{0} \\
\mathbf{v}_{i}
\end{array}\right], \hat{\boldsymbol{\$}}_{i 6}=\left[\begin{array}{c}
\mathbf{0} \\
\mathbf{z}_{i 1}
\end{array}\right] \\
& \hat{\boldsymbol{\$}}_{i 7}=\left[\begin{array}{c}
\mathbf{x}_{i 2} \\
\mathbf{c}_{i} \times \mathbf{x}_{i 2}
\end{array}\right], \hat{\boldsymbol{\$}}_{i 8}=\hat{\boldsymbol{\$}}_{i C}, \hat{\boldsymbol{\$}}_{i 9}=\left[\begin{array}{c}
\mathbf{z}_{i 2} \\
\mathbf{c}_{i} \times \mathbf{z}_{i 2}
\end{array}\right] \\
& \hat{\boldsymbol{\$}}_{i 10}=\left[\begin{array}{c}
\mathbf{0} \\
\mathbf{x}_{i 2}
\end{array}\right], \hat{\boldsymbol{\$}}_{i 11}=\left[\begin{array}{c}
\mathbf{0} \\
\mathbf{w}_{i}
\end{array}\right], \hat{\boldsymbol{\$}}_{i 12}=\left[\begin{array}{c}
\mathbf{0} \\
\mathbf{z}_{i 2}
\end{array}\right]
\end{aligned}
$$

where $\mathbf{J}_{\theta}^{i}$ considers the elastic deformation, while $\mathbf{J}_{q}^{i}$ takes into account the passive joint influence on the MP motions. Vectors $\mathbf{b}_{i}$ and $\mathbf{c}_{i}$, respectively, are the position vectors of points $B_{i}$ and $C_{i}$ in the reference frame. The corresponding unit vectors are given as

$$
\begin{aligned}
& \mathbf{z}_{i 1}=\frac{\mathbf{u}_{i} \times \mathbf{v}_{i}}{\left\|\mathbf{u}_{i} \times \mathbf{v}_{i}\right\|}, \mathbf{z}_{i 2}=\frac{\mathbf{v}_{i} \times \mathbf{w}_{i}}{\left\|\mathbf{v}_{i} \times \mathbf{w}_{i}\right\|} \\
& \mathbf{x}_{i 1}=\frac{\mathbf{v}_{i} \times \mathbf{z}_{i 1}}{\left\|\mathbf{v}_{i} \times \mathbf{z}_{i 1}\right\|}, \mathbf{x}_{i 2}=\frac{\mathbf{w}_{i} \times \mathbf{z}_{i 2}}{\left\|\mathbf{w}_{i} \times \mathbf{z}_{i 2}\right\|}
\end{aligned}
$$


The Cartesian stiffness matrix $\mathbf{K}_{i}$ of the $i$ th leg is obtained from the following matrix, namely,

$$
\left[\begin{array}{cc}
\mathbf{K}_{i} & * \\
* & *
\end{array}\right]=\left[\begin{array}{cc}
\mathbf{S}_{\theta}^{i} & \mathbf{J}_{q}^{i} \\
\mathbf{J}_{q}^{i T} & \mathbf{0}_{2}
\end{array}\right]^{-1}
$$

where the block $\mathbf{S}_{\theta}^{i}=\mathbf{J}_{\theta}^{i}\left(\mathbf{K}_{\theta}^{i}\right)^{-1} \mathbf{J}_{\theta}^{i^{T}} \in \mathbb{R}^{6}$ describes the spring compliance relative to the reference point on the mobile platform. Matrix $\mathbf{K}_{\theta}^{i} \in \mathbb{R}^{13}$ is the stiffness matrix in joint space, which describes the stiffness of the virtual springs and actuation, taking the form:

$$
\mathbf{K}_{\theta}^{i}=\left[\begin{array}{ccc}
K_{a c t}^{i} & \mathbf{0}_{1 \times 6} & \mathbf{0}_{1 \times 6} \\
\mathbf{0}_{6} & \mathbf{K}_{L_{1}}^{i} & \mathbf{0}_{6} \\
\mathbf{0}_{6} & \mathbf{0}_{6} & \mathbf{K}_{L_{2}}^{i}
\end{array}\right]
$$

where $K_{a c t}^{i}$ describes the actuation stiffness corresponding to the $i$ th actuator, and $\mathbf{K}_{L_{1(2)}}^{i^{-1}} \in \mathbb{R}^{6}$ is the stiffness matrices of the lower (upper) curved links in the $i$ th leg. The compliance matrix $\mathbf{K}_{L_{1(2)}}^{i^{-1}}$ of the curved link was documented in Wu (2012); Wu et al. (2014):

$$
\mathbf{K}_{L_{1(2)}}^{i^{-1}}=\left[\begin{array}{cccccc}
C_{11} & C_{12} & 0 & 0 & 0 & C_{16} \\
C_{12} & C_{22} & 0 & 0 & 0 & C_{26} \\
0 & 0 & C_{33} & C_{34} & C_{35} & 0 \\
0 & 0 & C_{34} & C_{44} & C_{45} & 0 \\
0 & 0 & C_{35} & C_{45} & C_{55} & 0 \\
C_{16} & C_{26} & 0 & 0 & 0 & C_{66}
\end{array}\right]
$$

The Cartesian stiffness matrix $\mathbf{K}$ of the system is found by simple addition from Eqn. (9), namely,

$$
\mathbf{K}=\sum_{i=1}^{3} \mathbf{K}_{i}
$$

\section{Parameter Nomalization of the Stiffness Matrix}

The previous Cartesian stiffness matrix (12) is inhomogeneous, namely,

$$
\left[\begin{array}{c}
\mathbf{m} \\
\mathbf{f}
\end{array}\right]=\left[\begin{array}{cc}
\mathbf{K}_{r r}[\mathrm{Nm}] & \mathbf{K}_{r t}[\mathrm{~N}] \\
\mathbf{K}_{r t}^{T}[\mathrm{~N}] & \mathbf{K}_{t t}[\mathrm{~N} / \mathrm{m}]
\end{array}\right]\left[\begin{array}{c}
\Delta \varphi \\
\Delta \mathbf{p}
\end{array}\right]
$$

In order to analyze the stiffness matrix, it is necessary to make it homogeneous. Henceforth, the method reported by Kövecses and Ebrahimi (2009) is adopted to decompose the stiffness matrix $\mathbf{K}$ into dimensionally homogeneous entries. Equation (13) can be decomposed as

$$
\begin{aligned}
& \mathbf{m}=\mathbf{K}_{r r} \Delta \boldsymbol{\varphi}+\mathbf{K}_{r t} \Delta \mathbf{p}=\mathbf{m}_{r}+\mathbf{m}_{p} \\
& \mathbf{f}=\mathbf{K}_{r t}^{T} \Delta \boldsymbol{\varphi}+\mathbf{K}_{t t} \Delta \mathbf{p}=\mathbf{f}_{r}+\mathbf{f}_{p}
\end{aligned}
$$

In Eqn. (14a), the two unit-homogeneous parts can be associated with a physically meaningful quadratic form, for instance, $\mathbf{m}_{r}^{T} \mathbf{m}_{r}=\Delta \boldsymbol{\varphi}^{T} \mathbf{K}_{r r}^{T} \mathbf{K}_{r r} \Delta \boldsymbol{\varphi}$, which defines a moment ellipsoid in the Cartesian space. The eigenvectors of $\mathbf{K}_{r r}^{T} \mathbf{K}_{r r}$ define the principal axes of the ellipsoid, which is related to the singular values obtained through singular value decomposition (SVD) of matrix $\mathbf{K}_{r r}$, namely,

$$
\mathbf{K}_{r r}=\mathbf{U}_{r r} \boldsymbol{\Sigma}_{r r} \mathbf{V}_{r r}^{*}
$$

where $\boldsymbol{\Sigma}_{r r}$ is a diagonal matrix composed of the singular values of $\mathbf{K}_{r r}$ indicating the principal axes of the ellipsoid and the columns of matrix $\mathbf{U}_{r r}$ are the eigenvectors of matrix $\mathbf{K}_{r r} \mathbf{K}_{r r}^{T}$ indicating the directions of the principal axes. The symmetric $3 \times 3$ matrix $\mathbf{K}_{r r}^{T} \mathbf{K}_{r r}$ has three orthogonal eigenvectors $\mathbf{v}_{r 1}, \mathbf{v}_{r 2}$ and $\mathbf{v}_{r 3}$ with dimensions of $\Delta \boldsymbol{\varphi} . \Delta \boldsymbol{\varphi}$ can be represented by a dimensionless vector $\mathbf{s}_{r, m}$ through linear transformation Kövecses and Ebrahimi (2009), namely,

$$
\Delta \varphi=\mathbf{V}_{r r} \mathbf{s}_{r, m}, \mathbf{V}_{r r}=\left[\begin{array}{lll}
\mathbf{v}_{r 1} & \mathbf{v}_{r 2} & \mathbf{v}_{r 3}
\end{array}\right]
$$

Similarly, $\Delta \mathbf{p}$ can be expressed as

$$
\Delta \mathbf{p}=\mathbf{V}_{r t} \mathbf{s}_{p, m}, \mathbf{V}_{r t}=\left[\begin{array}{lll}
\mathbf{v}_{t 1} & \mathbf{v}_{t 2} & \mathbf{v}_{t 3}
\end{array}\right]
$$

where $\mathbf{v}_{t i}, i=1,2,3$, are the eigenvectors of matrix $\mathbf{K}_{r t}^{T} \mathbf{K}_{r t}$. By the same token, $\Delta \varphi$ and $\Delta \mathbf{p}$ from Eqn. (14b) can be expressed as

$$
\Delta \varphi=\mathbf{V}_{t r} \mathbf{s}_{r, f}, \Delta \mathbf{p}=\mathbf{V}_{t t} \mathbf{s}_{p, f}
$$

where $\mathbf{V}_{t r}$ and $\mathbf{V}_{t t}$ are orthogonal matrices composed of the eigenvectors of matrices $\mathbf{K}_{r t} \mathbf{K}_{r t}^{T}$ and $\mathbf{K}_{t t}^{T} \mathbf{K}_{t t}$, respectively. Substituting Eqns. (16), (17) and (18) into Eqns. (14a) and (14b), respectively, leads to

$$
\begin{aligned}
& \mathbf{m}=\mathbf{K}_{r r} \mathbf{V}_{r r} \mathbf{s}_{r, m}+\mathbf{K}_{r t} \mathbf{V}_{r t} \mathbf{s}_{p, m}=\mathbf{G}_{m}\left[\begin{array}{cc}
\mathbf{s}_{r, m}^{T} & \mathbf{s}_{p, m}^{T}
\end{array}\right]^{T} \\
& \mathbf{f}=\mathbf{K}_{r t}^{T} \mathbf{V}_{t r} \mathbf{s}_{r, f}+\mathbf{K}_{t t} \mathbf{V}_{t t} \mathbf{s}_{p, f}=\mathbf{G}_{f}\left[\begin{array}{ll}
\mathbf{s}_{r, f}^{T} & \mathbf{s}_{p, f}^{T}
\end{array}\right]^{T}
\end{aligned}
$$

where $\mathbf{G}_{m}$ and $\mathbf{G}_{f}$ are defined as

$$
\begin{aligned}
& \mathbf{G}_{m}=\left[\begin{array}{ll}
\mathbf{K}_{r r} \mathbf{V}_{r r} & \mathbf{K}_{r t} \mathbf{V}_{r t}
\end{array}\right] \\
& \mathbf{G}_{f}=\left[\begin{array}{ll}
\mathbf{K}_{r t}^{T} \mathbf{V}_{t r} & \mathbf{K}_{t t} \mathbf{V}_{t t}
\end{array}\right]
\end{aligned}
$$

$\mathbf{G}_{m}$ and $\mathbf{G}_{f}$ are $3 \times 6$ dimensionally homogeneous matrices, mapping the normalized displacements to the vector of moments (forces). The SVD of matrix $\mathbf{G}_{m}\left(\mathbf{G}_{f}\right)$ indicates the rotational (translational) stiffness property of the manipulator at a prescribed posture. Matrix $\mathbf{G}_{m}\left(\mathbf{G}_{f}\right)$ has three nonzero singular values $\sigma_{j, r}\left(\sigma_{j, t}\right), j=1,2,3$, which characterize the distortion of the unit sphere $\|\mathbf{m}\|=1 \mathrm{Nm}(\|\mathbf{f}\|=1 \mathrm{~N})$ as 


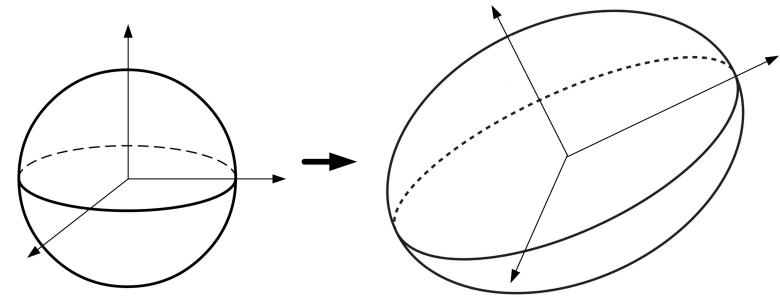

Figure 5: Distortion of the normalized moment/force sphere.

shown in Fig. 5. Consequently, the rotational stiffness index can be defined Taghvaeipour et al. (2012) as

$$
\kappa_{r}=\min \left\{\sigma_{j, r}\right\}, j=1,2,3
$$

Similarly, the translational stiffness index is defined as

$$
\kappa_{t}=\min \left\{\sigma_{j, t}\right\}
$$

The two performance indices $\kappa_{r}$ and $\kappa_{t}$ assess the stiffness behavior of the manipulator, a higher index indicating high rigidity.

These singular values can be graphically represented by ellipsoids to indicate the translational and rotational stiffness, respectively, where the principal axes of the ellipsoid indicate the singular values and their directions indicate the corresponding left singular vectors. The isotropy of the stiffness can be measured by the ratio of $\rho=\sigma_{\min } / \sigma_{\max } \in[0,1]$, namely, the inverse of the conditioning number of the matrices $\mathbf{G}_{m}\left(\mathbf{G}_{f}\right)$.

\section{Stiffness Optimization and Analysis of the Co-axial SPM}

The foregoing approaches are applied to the SPM in Fig. 1, for which only the upper curved links are needed to be considered. The actuation stiffness is obtained as $K_{a c t}^{i}=5.44 \cdot 10^{5} \mathrm{Nm} / \mathrm{rad}$ from the static load experiment implemented on an actuator with gearbox as shown in Fig. 6, where the output shaft was mechanically locked. The curved link of midcurve radius $R$ with circular cross-section of radius $r$, is made of steel of Young's modulus $E=210 \mathrm{GPa}$, Poisson's ratio $\nu=0.3$ and the shear modulus $G=E /(2+2 \nu)$. Moreover, the SPM is assembled under the working mode Bonev et al. (2006) of $\left(\mathbf{u}_{i} \times \mathbf{v}_{i}\right) \cdot \mathbf{w}_{i} \leq 0, i=1,2,3$.

\subsection{SPM Stiffness Optimization}

The manipulator stiffness heavily depends on its geometric and structural parameters, thus, the optimiza-

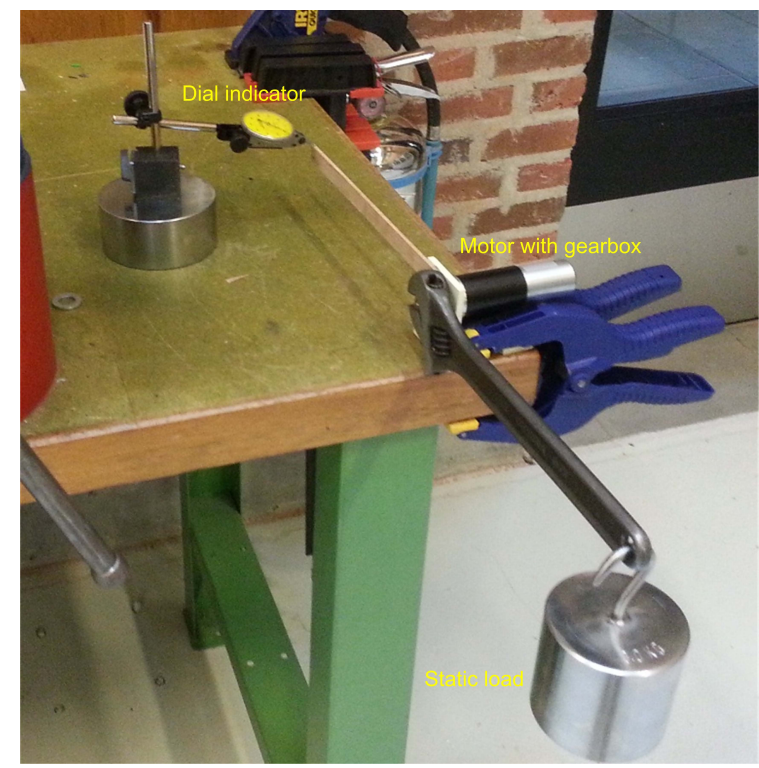

Figure 6: Experimental setup of the actuation stiffness measurement.

tion method could be used to find the optimal parameters. Here, the radius of the cross-section of the curved link is $r=7.5 \mathrm{~mm}$, and the design variables in this optimization problem are:

$$
\mathbf{x}=\left[\begin{array}{llll}
\alpha_{1} & \alpha_{2} & \beta & R
\end{array}\right]^{T}
$$

Henceforth, the workspace of the SPM described in Fig. 3 is specified as $\phi \in \mathcal{S}, \sigma \in \mathcal{S}, \theta \in\left[0, \theta_{\text {min }}\right]$, where $\mathcal{S}=(-\pi, \pi], \theta_{\min } \geq 45^{\circ}$, subsequently, the design space can be found as:

$$
\alpha_{1} \in\left[45^{\circ}, 90^{\circ}\right] ; \alpha_{2} \in\left[45^{\circ}, 135^{\circ}\right] ; \beta \in\left[45^{\circ}, 90^{\circ}\right]
$$

By the analysis above, the index $\kappa_{r} \kappa_{t}$ is defined to evaluate the SPM stiffness, consequently, the objective function is written as:

$$
f(\mathbf{x})=\min \left(\kappa_{n, r} \kappa_{n, t}\right) \quad \rightarrow \quad \max
$$

Here, the index is calculated through a discrete approach, which is carried out over $n=n_{1} \times n_{2} \times n_{3}$ workspace points, where $n_{1}, n_{2}$ and $n_{3}$ are the numbers of discrete points on $\phi, \theta$ and $\sigma$, respectively. Mathematically, the optimization problem for the SPM is formulated as:

$$
\begin{aligned}
\operatorname{maxmize} & f(\mathbf{x})=\min \left(\kappa_{n, r} \kappa_{n, t}\right) \\
\text { over } & \mathbf{x}=\left[\begin{array}{llll}
\alpha_{1} & \alpha_{2} & \beta & R
\end{array}\right]^{T} \\
\text { subject to } & g_{1}: 120 \mathrm{~mm} \leq R \leq 300 \mathrm{~mm} \\
& g_{2}: R \sin \alpha_{1} \geq 120 \mathrm{~mm} \\
& g_{3}: 45^{\circ} \leq\left\{\alpha_{1}, \beta\right\} \leq 90^{\circ} \\
& g_{4}: 45^{\circ} \leq \alpha_{2} \leq 135^{\circ}
\end{aligned}
$$




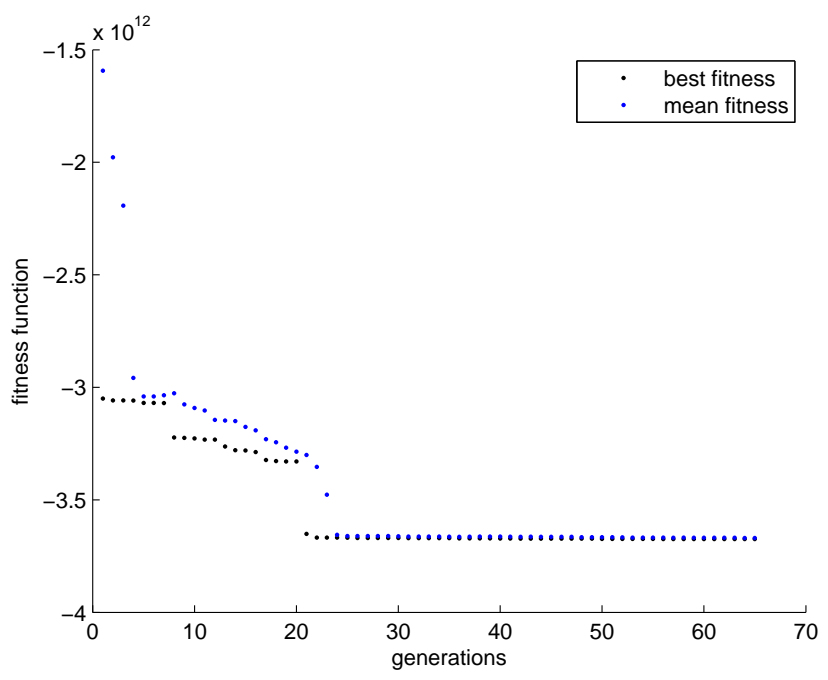

Figure 7: Convergence for the stiffness optimization problem of the SPM.

This optimization procedure was implemented with the MATLAB genetic algorithm (GA) toolbox. Here, the initial variables, with population size 200, were randomly generated. Figure 7 shows the optimization results. After 65 generations, the objective function converged, for which the best solution is found as:

$$
\mathbf{x}_{b}=\left[\begin{array}{llll}
55^{\circ} & 86^{\circ} & 85^{\circ} & 157.5 \mathrm{~mm}
\end{array}\right]^{T}
$$

Optimization problem (26) aims to maximize both the rotational and the translational stiffness. In practice, index $\kappa_{r}$ or $\kappa_{t}$ can be specified as the objective corresponding to the manipulator applications.

\subsection{SPM Stiffness Analysis}

With the optimal design variables (27), at configurations with tilt angle $\theta=0$, the blocks in the stiffness matrix of (12) are constantly equal to

$$
\begin{aligned}
\mathbf{K}_{r r}= & {\left[\begin{array}{ccc}
0.0101 & 0 & 0 \\
0 & 0.0101 & 0 \\
0 & 0 & 0.0383
\end{array}\right] \cdot 10^{7} } \\
\mathbf{K}_{r t}= & {\left[\begin{array}{ccc}
-0.0794 & 0.0673 & 0 \\
-0.0673 & -0.0794 & 0 \\
0 & 0 & 0.1587
\end{array}\right] \cdot 10^{7} } \\
\mathbf{K}_{t t}= & {\left[\begin{array}{ccc}
1.1594 & 0 & 0 \\
0 & 1.1594 & 0 \\
0 & 0 & 0.6966
\end{array}\right] \cdot 10^{7} }
\end{aligned}
$$

where, the blocks $\mathbf{K}_{r r}, \mathbf{K}_{r t}$ and $\mathbf{K}_{t t}$ are given in $\mathrm{Nm} / \mathrm{rad}, \mathrm{N} / \mathrm{rad}$ and $\mathrm{N} / \mathrm{m}$, respectively.

According to the decomposition presented in Sec. 4, the three singular values and vectors corresponding to the rotational stiffness are found as:

$$
\begin{aligned}
& {[\boldsymbol{\sigma}]_{r}=\left[\begin{array}{lll}
1.6328 & 1.0457 & 1.0457
\end{array}\right] \cdot 10^{6} \mathrm{Nm}} \\
& {[\mathbf{v}]_{r}=\left[\begin{array}{ccc}
\left(\begin{array}{c}
0 \\
0 \\
-1
\end{array}\right) & \left(\begin{array}{c}
-1 \\
0 \\
0
\end{array}\right) & \left(\begin{array}{c}
0 \\
-1 \\
0
\end{array}\right)
\end{array}\right]}
\end{aligned}
$$

Similarly, the singular values and vectors for translational stiffness are:

$$
\begin{aligned}
& {[\boldsymbol{\sigma}]_{t}=\left[\begin{array}{lll}
1.1640 & 1.1640 & 0.7144
\end{array}\right] \cdot 10^{7} \mathrm{~N}} \\
& {[\mathbf{v}]_{t}=\left[\begin{array}{ccc}
\left(\begin{array}{c}
-1 \\
0 \\
0
\end{array}\right) & \left(\begin{array}{c}
0 \\
-1 \\
0
\end{array}\right) & \left(\begin{array}{l}
0 \\
0 \\
1
\end{array}\right)
\end{array}\right]}
\end{aligned}
$$

It is noted the decomposed vectors, i.e., the directions of rotational/translational stiffness, are parallel to the original frame axes.

On the basis of foregoing analysis, the corresponding rotational and translational stiffness ellipsoids are visualized in Fig. 8. It is found that the rotational stiffness formulates a prolate spheroid while the translational stiffness in the shape of an oblate spheroid. This means that at configurations with tilt angle $\theta=0$, the SPM has the largest rotational stiffness about $z$-axis but the smallest translational stiffness along $z$-axis; in the $x y$ quadrant, it has the same rotational/translational stiffness in all the directions. In other words, the elastic deformations of the manipulator under a prescribed moment will formulate oblate spheroids, namely, the directions of semi-minor/-major axes of the stiffness ellipsoids are parallel to the directions of the semimajor/-minor axes of the deformation ellipsoids. Figure 9 displays the deformation ellipsoids when a moment $\|\mathbf{m}\|=10 \mathrm{Nm}$ acting on the mobile platform, which shows that the elastic displacements reach to their minimum magnitudes when the direction of the moment is about $z$-axis.

The previously defined indices $\kappa_{r}$ and $\kappa_{t}$ mapping over the regular workspace with $\sigma=0$ are shown in Fig. 10, from which it is seen that the SPM stiffness distribution is rotationally symmetrical with the interval of $120^{\circ}$ that associates with its symmetrical structure. At the home configuration with $\theta=0$ and the workspace boundaries of $\phi=-100^{\circ}, 20^{\circ}$ and $140^{\circ}, \kappa_{r}$ reaches to the maximum value. The minimum $\kappa_{r}$ appears at the workspace bounds at $\phi=-165^{\circ},-45^{\circ}$ and $75^{\circ}$. Both the maximum and the minimum $\kappa_{t}$ occur at the workspace boundary, the maximum values appearing at $\phi=-170^{\circ},-50^{\circ}$ and $70^{\circ}$ while the minimum ones at $\phi=-100^{\circ}, 20^{\circ}$ and $140^{\circ}$. The isotropy of the stiffness, namely, the distributions of the inverse of the condition number of the stiffness matrix are given in Figs. 10(c) and 10(d). By comparison with Figs. 10(a) and $10(\mathrm{~b})$, when the SPM has relatively larger rota- 
tional/translational stiffness, the manipulator stiffness behaves relatively more isotropic.

The stiffness ellipsoids at two representative configurations, where the SPM has the minimum rotational/translational stiffness, are displayed in Fig. 11, respectively, graphically indicating the directions of the weak stiffness. At orientation $\left[75^{\circ}, 45^{\circ}, 0\right]$, the singular values and vectors are found as below:

$$
\begin{aligned}
& {[\boldsymbol{\sigma}]_{r}=\left[\begin{array}{lll}
1.6544 & 1.4266 & 0.7119
\end{array}\right] \cdot 10^{6} \mathrm{Nm}} \\
& \left.[\mathbf{v}]_{r}=\left[\begin{array}{c}
-0.4201 \\
-0.6968 \\
-0.5813
\end{array}\right) \quad\left(\begin{array}{c}
0.5384 \\
0.3243 \\
-0.7778
\end{array}\right) \quad\left(\begin{array}{c}
0.7305 \\
-0.6397 \\
0.2389
\end{array}\right)\right] \\
& {[\boldsymbol{\sigma}]_{t}=\left[\begin{array}{lll}
1.7011 & 0.8673 & 0.7509
\end{array}\right] \cdot 10^{7} \mathrm{~N}} \\
& \left.[\mathbf{v}]_{t}=\left[\begin{array}{c}
-0.4910 \\
0.5967 \\
-0.6347
\end{array}\right) \quad\left(\begin{array}{l}
0.4720 \\
0.7946 \\
0.3819
\end{array}\right) \quad\left(\begin{array}{c}
0.7322 \\
-0.1120 \\
-0.6718
\end{array}\right)\right]
\end{aligned}
$$

In comparison with the stiffness ellipsoids at orientation $\theta=0$, the ratio of the semi-minor/-major axes, i.e., the stiffness isotropy, is much smaller, however, the volumes of the ellipsoids are quite close. At orientation $\left[20^{\circ}, 45^{\circ}, 0\right]$, the manipulator stiffness formulates the similar shaped ellipsoids.

Due to the symmetrical structure, the stiffness distributions and isotropy with different values of $\sigma$ can be obtained with the rotation of the isocontours in Fig. 10. As a consequence, it can be predicted that the global stiffness of the SPM becomes weak towards the workspace boundary from the center region.

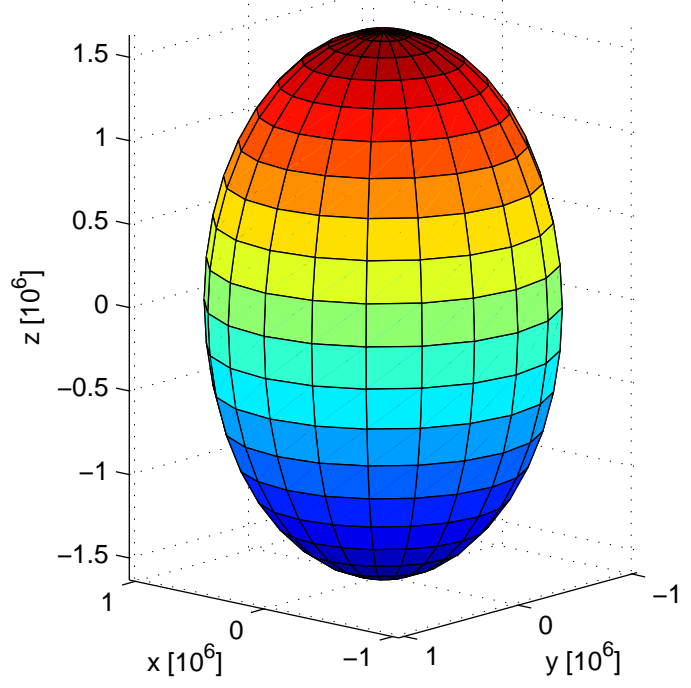

(a)

\section{Conclusions}

In this paper, the stiffness characteristics of the spherical parallel manipulators is discussed. The Cartesian stiffness matrix is formulated by considering both the rotational and the translational deflection. By means of singular value decomposition, the dimensionally inhomogeneous stiffness matrix is transformed into two homogeneous ones, corresponding to the force and moment equations. The stiffness properties of a manipulator at a given configuration can be characterized through singular value decomposition of these two homogeneous matrices, which indicates the directions of the high/weak stiffness.

Two performance indices for rotational/translational stiffness were introduced to evaluate the manipulator stiffness throughout the Cartesian workspace. The results can be used for either assessment of the stiffness at different configurations or design optimization. The proposed approach is illustrated by a case study of a co-axial SPM with unlimited-rolling motion, whose isocontours of the rotational and translational stiffness indices within a regular workspace were mapped together with the isotropy. The maps show that the SPM is stiffer in the center region of its workspace and show an image of the SPM overall stiffness.

\section{References}

Angeles, J. On the nature of the Cartesian stiffness matrix. Ingeniería Mecánica, 2010. 3(5):163-170.

Asada, H. and Granito, J. Kinematic and static char-

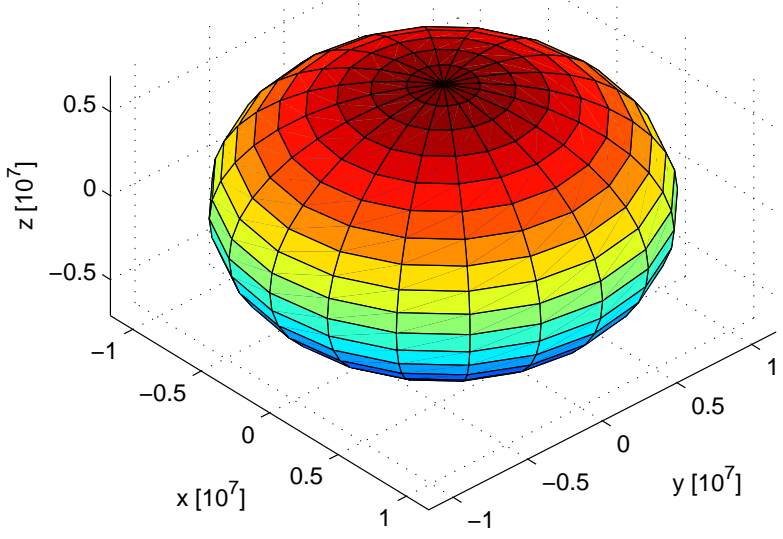

(b)

Figure 8: Graphical representation of the SPM stiffness ellipsoid with $\theta=0$ : (a) $\sigma_{r}$; (b) $\sigma_{t}$. 


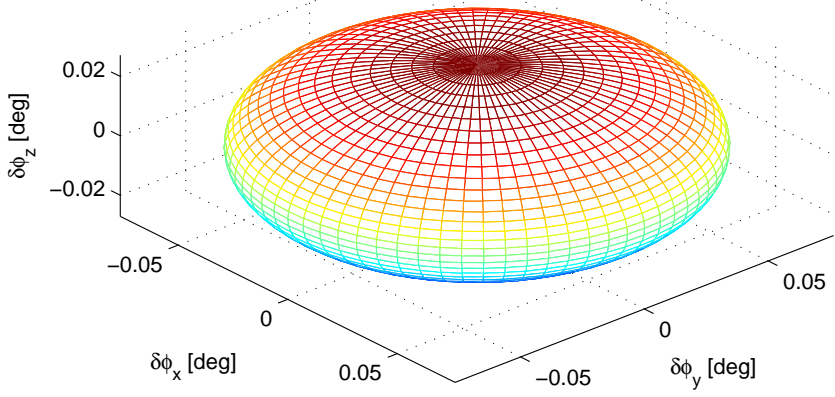

(a)

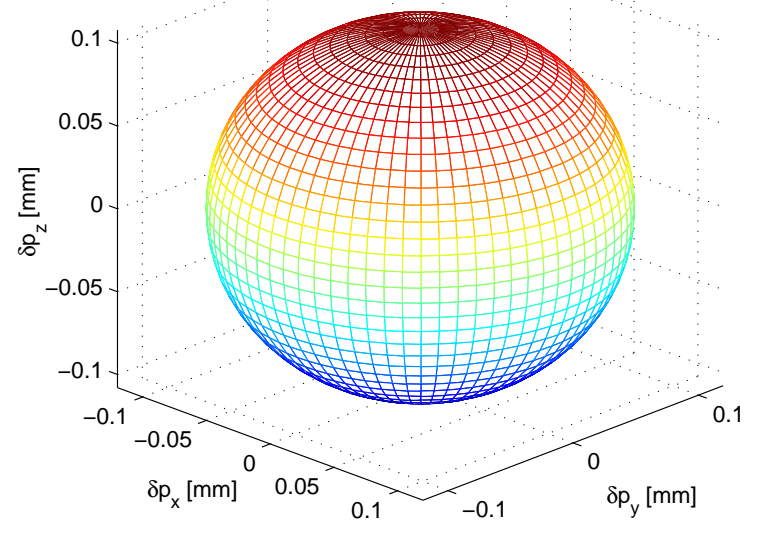

(b)

Figure 9: Deformation ellipsoid with orientation of $\theta=0$ under a pure moment $\|\mathbf{m}\|=10 \mathrm{Nm}$ : (a) orientation error; (b) translational deformation.

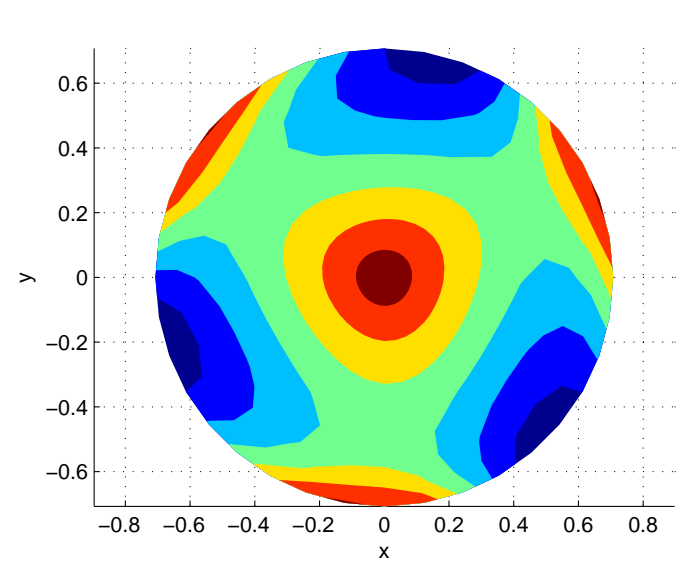

(a)

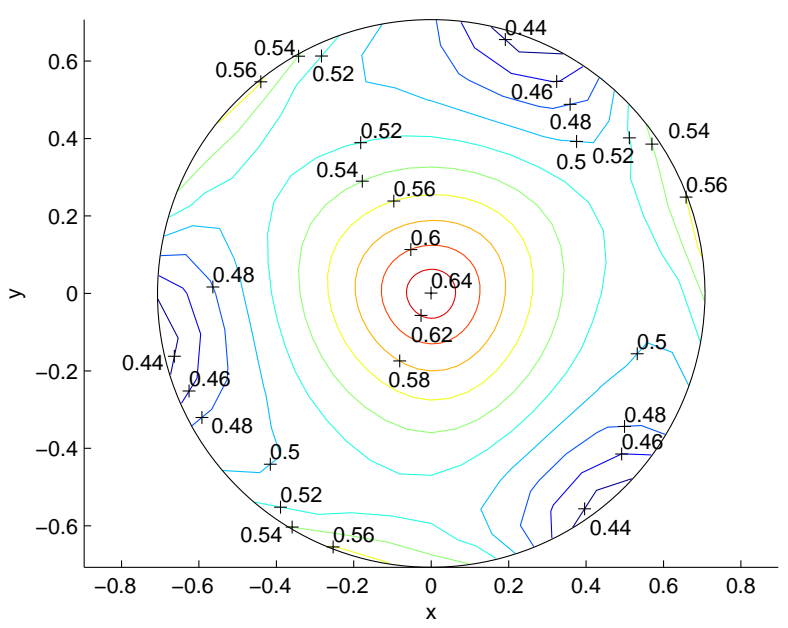

(c)

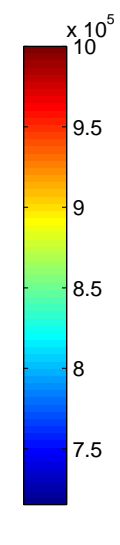

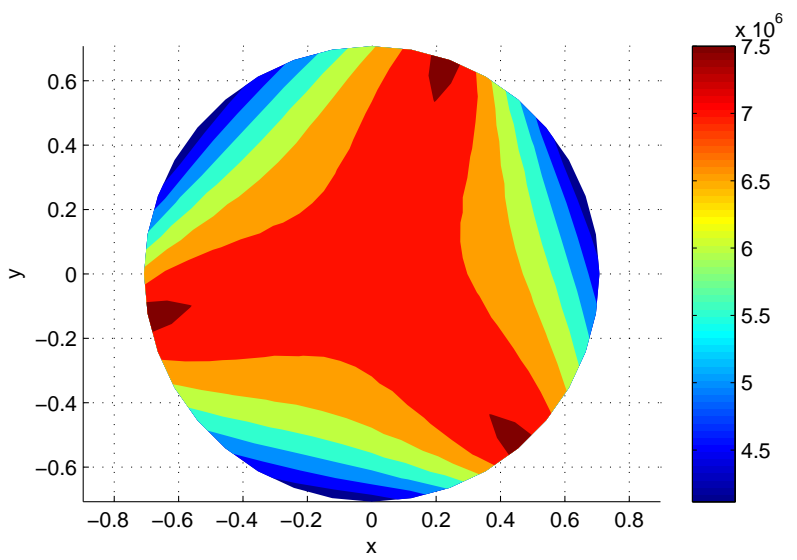

(b)

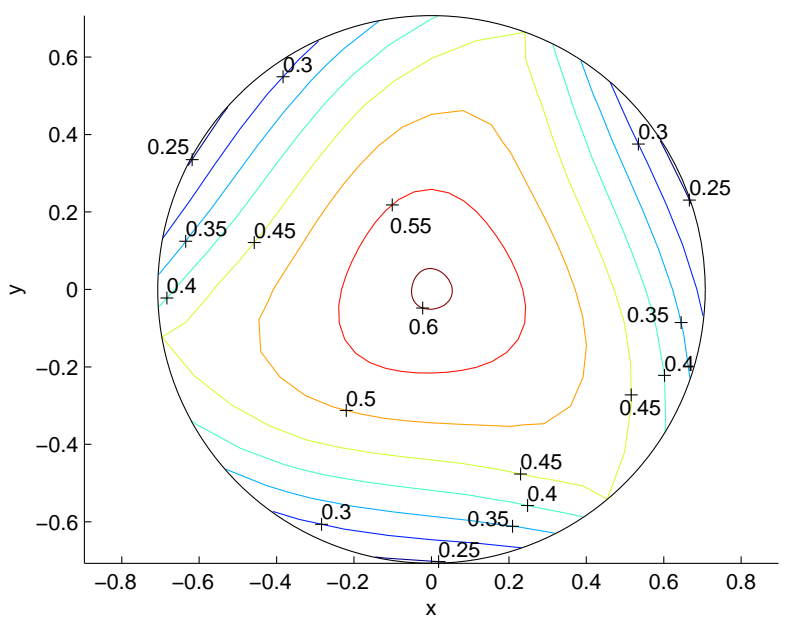

(d)

Figure 10: The distribution of the stiffness indices and isotropy over the regular workspace with torsion angle $\sigma=0$ : (a) $\kappa_{r} ;$ (b) $\kappa_{t}$; (c) $\rho_{r}$; (b) $\rho_{t}$. 


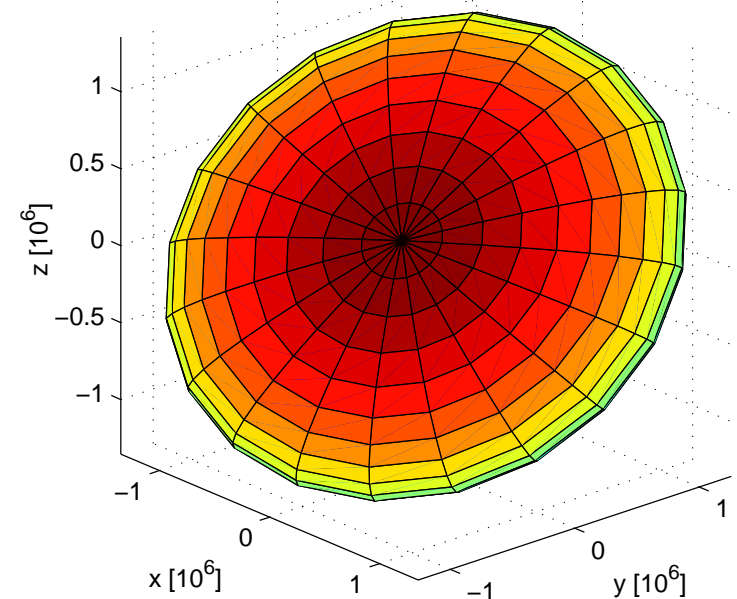

(a) $\sigma_{r}$

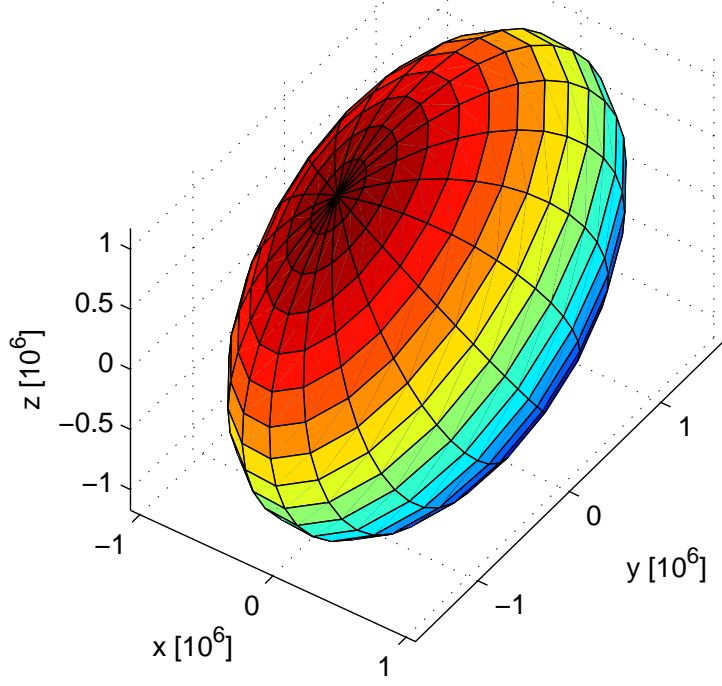

(c) $\sigma_{r}$

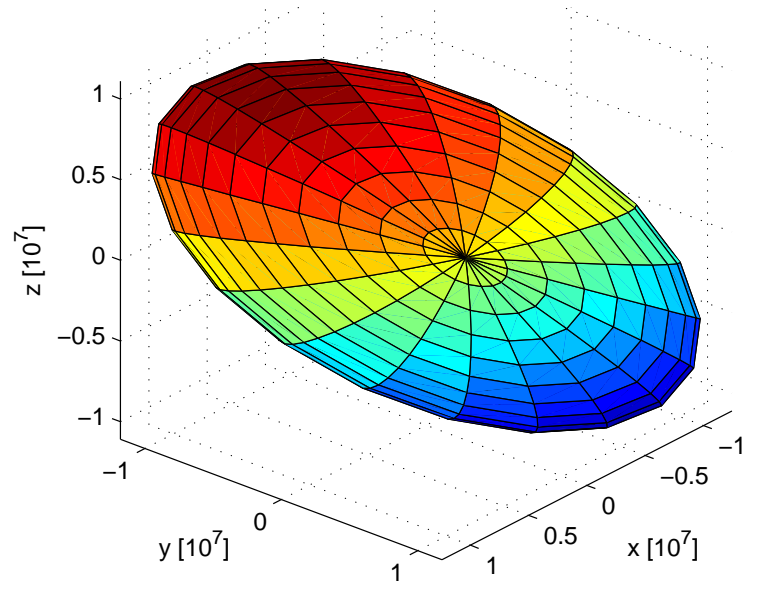

(b) $\sigma_{t}$

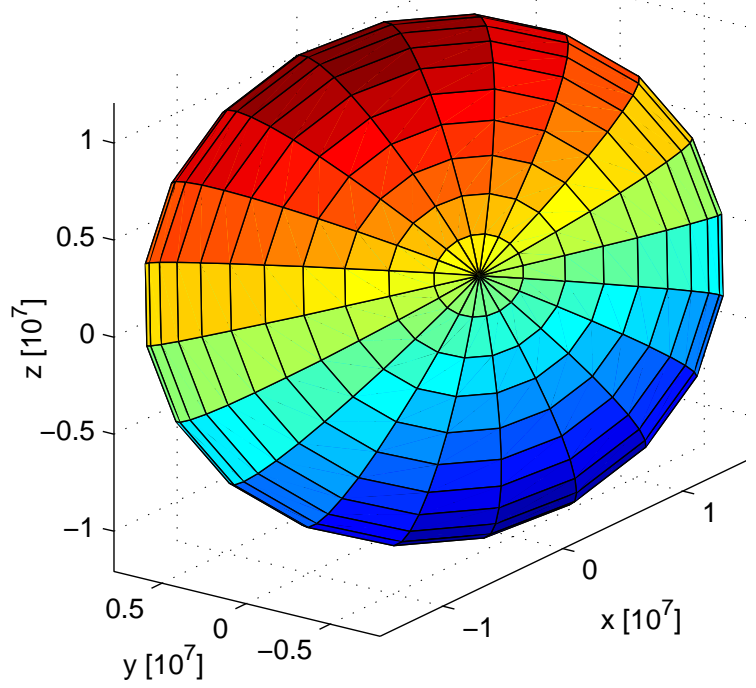

(d) $\sigma_{t}$

Figure 11: Stiffness ellipsoids: (a) and (b) at orientation $\left[75^{\circ}, 45^{\circ}, 0\right]$ when the SPM has the minimum rotational stiffness; (c) and (d) at orientation $\left[20^{\circ}, 45^{\circ}, 0\right]$ when the SPM has the minimum translational stiffness. 
acterization of wrist joints and their optimal design. In IEEE International Conference on Robotics and Automation, volume 2. pages 244-250, 1985. doi:10.1109/ROBOT.1985.1087324.

Bai, S. Optimum design of spherical parallel manipulators for a prescribed workspace. Mechanism and Machine Theory, 2010. 45(2):200-211. doi:10.1016/j.mechmachtheory.2009.06.007.

Bai, S., Hansen, M. R., and Andersen, T. O. Modelling of a special class of spherical parallel manipulators with Euler parameters. Robotica, 2009. 27(2):161170. doi:10.1017/S0263574708004402.

Bonev, I. Direct kinematics of zero-torsion parallel mechanisms. In IEEE International Conference on Robotics and Automation. Pasadena, California, USA, pages 3851-3856, 2008. doi:10.1109/ROBOT.2008.4543802.

Bonev, I. A., Chablat, D., and Wenger, P. Working and assembly modes of the Agile Eye. In International Conference on Robotics and Automation. pages 2317-2322, 2006. doi:10.1109/ROBOT.2006.1642048.

Bonev, I. A. and Gosselin, C. M. Singularity loci of spherical parallel mechanisms. In IEEE International Conference on Robotics and Automation. pages 2957-2962, 2005. doi:10.1109/ROBOT.2005.1570563.

Bulca, F., Angeles, J., and Zsombor-Murray, P. J. On the workspace determination of spherical serial and platform mechanisms. Mechanism and Machine Theory, 1999. 34(3):497-512. doi:10.1016/S0094114X(98)00019-6.

Ciblak, N. and Lipkin, H. Synthesis of Cartesian stiffness for robotic applications. In IEEE International Conference on Robotics and Automation, volume 3. pages 2147-2152, 1999. doi:10.1109/ROBOT.1999.770424.

Dai, J. and Ding, X. Compliance analysis of a three-legged rigidly-connected platform device. Journal of Mechanical Design, 2006. 128. doi:10.1115/1.2202141.

Ding, X. and Selig, J. M. On the compliance of coiled springs. International Journal of Mechanical Sciences, 2004. 46(5):703-727. doi:10.1016/j.ijmecsci.2004.05.009.

El-Khasawneh, B. S. and Ferreira, P. M. Computation of stiffness and stiffness bounds for parallel link manipulators. International Journal of $\mathrm{Ma}$ chine Tools and Manufacture, 1999. 39(2):321-342. doi:10.1016/S0890-6955(98)00039-X.

Enferadi, J. and Tootoonchi, A. A. Accuracy and stiffness analysis of a 3-RRP spherical parallel manipulator. Robotica, 2011. 29:193-209. doi:10.1017/S0263574710000032.

Gosselin, C. Stiffness mapping for parallel manipulators. IEEE Transactions on Robotics and Automation, 1990. 6(3):377-382. doi:10.1109/70.56657.

Gosselin, C. M. and Lavoie, E. On the kinematic design of spherical three-degree-of-freedom parallel manipulators. The International Journal of Robotics Research, 1993. 12(4):394-402. doi:10.1177/027836499301200406.

Kong, X. and Gosselin, C. M. Type synthesis of three-degree-of-freedom spherical parallel manipulators. The International Journal of Robotics Research, 2004. 23(3):237-245. doi:10.1177/0278364904041562.

Kövecses, J. and Ebrahimi, S. Parameter analysis and normalization for the dynamics and design of multibody systems. Journal of Computational and Nonlinear Dynamics, 2009. 4(3):031008(1-10). doi:10.1115/1.2202141.

Liu, X. J., Jin, Z. L., and Gao, F. Optimum design of 3-DOF spherical parallel manipulators with respect to the conditioning and stiffness indices. Mechanism and Machine Theory, 2000. 35(9):1257-1267. doi:10.1016/S0094-114X(99)00072-5.

Pashkevich, A., Chablat, D., and Wenger, P. Stiffness analysis of overconstrained parallel manipulators. Mechanism and Machine Theory, 2009. 44(5):966982. doi:10.1016/j.mechmachtheory.2008.05.017.

Taghvaeipour, A., Angeles, J., and Lessard, L. On the elastostatic analysis of mechanical systems. Mechanism and Machine Theory, 2012. 58:202-216. doi:10.1016/j.mechmachtheory.2012.07.011.

$\mathrm{Wu}, \mathrm{G}$. Multiobjective optimum design of a 3RRR spherical parallel manipulator with kinematic and dynamic dexterities. Modeling, Identification and Control, 2012. 33(3):111-122. doi:10.4173/mic.2012.3.3.

Wu, G., Bai, S., and Kepler, J. Mobile platform center shift in spherical parallel manipulators with flexible limbs. Mechanism and Machine Theory, 2014. 75:12-26. doi:10.1016/j.mechmachtheory.2014.01.001. 